\title{
60-GHz CPW-Fed Post-Supported Patch Antenna Using Micromachining Technology
}

Jeong-Geun Kim, Hyung Suk Lee, Ho-Seon Lee, Jun-Bo Yoon, Member, IEEE, and Songcheol Hong, Member, IEEE

\begin{abstract}
A 60-GHz coplanar waveguide (CPW)-fed post-supported patch antenna is presented using micromachining technology. In the proposed structure, the radiating patch and the feed line network can be optimized separately with a substrate. The antenna performance is improved by elevating the patch in the air. A patch array antenna is also designed with a simple feed network. The fabricated antenna shows broad band characteristics such as $-10 \mathrm{~dB}$ bandwidth of $4.3 \mathrm{GHz}$ from $58.7 \mathrm{GHz}$ to $64.5 \mathrm{GHz}$ in the single patch antenna and $8.7 \mathrm{GHz}$ from $56.3 \mathrm{GHz}$ to $65 \mathrm{GHz}$ in $2 \times 1$ patch array antenna.
\end{abstract}

Index Terms-Coplanar waveguide (CPW)-fed post-supported antenna, micromachined antenna, 60-GHz WLAN antenna.

\section{INTRODUCTION}

A S THE demands of high data rates are increased, millimeter wave communication systems such as $60-\mathrm{GHz}$ wireless local-area networks (LANs) have been getting a lot of attention [1]-[3]. At mm-wave frequency, horn antenna is generally used due to its high performance, but it is very bulky, heavy, and high cost. Moreover, the transition of waveguideto-microstrip transition is needed to interconnect with monolithic microwave integrated circuits (MMICs). It is difficult to fabricate the transition, as the additional losses of the transition reduce the transmitting output power and degrade the noise figure of the receiver directly because the transition is in front of the low noise amplifier (LNA). Microstrip patch antenna is widely used at low frequency on a printed circuit board (PCB) substrate because it can be implemented with low profile, low weight, and low cost. However, it shows poor performance due to high substrate losses and low radiation efficiency at mm-wave frequency. Thus, it can not be applied well at mm-wave frequency.

Recently, as micromachining technology is developed, it is applied to the antenna to improve the performance [4]-[7]. Usually, two kinds of MEMS antenna structures are reported. One is a MEMS antenna supported with a thin membrane, the other is a MEMS aperture coupled antenna. In the case of a membrane supported patch antenna, because the substrate under the patch is etched out, the substrate losses of the antenna can be reduced. Also, since the dielectric constant under the patch is

Manuscript received December 16, 2004; revised June 6, 2005. This work was supported by KOSEF under the ERC Program through the MINT Research Center, Dongguk University. The review of this letter was arranged by Associate Editor A. Weisshaar.

The authors are with the Department of Electrical Engineering, Korea Advanced Institute of Science and Technology, Daejeon 305-701, Korea (e-mail: junggun@eeinfo.kaist.ac.kr).

Digital Object Identifier 10.1109/LMWC.2005.856690

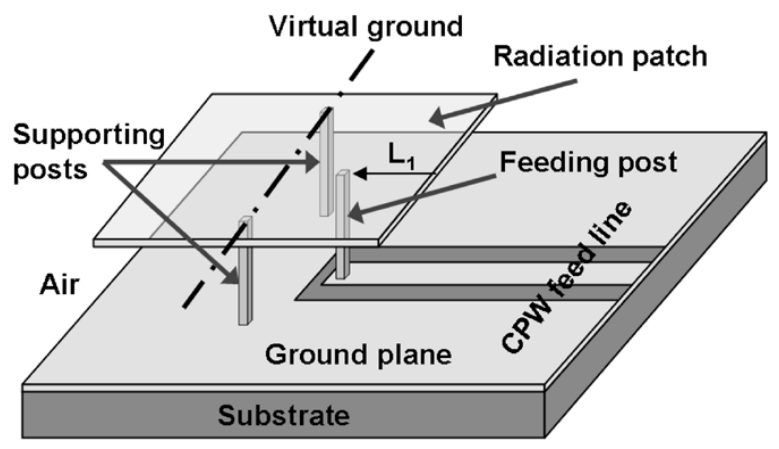

Fig. 1. Proposed CPW-fed post-supported MEMS antenna.

almost the same with the air, the radiation efficiency can be improved. However, the delicate substrate etching has to be performed because the substrate etching affects the antenna performance seriously at mm-wave frequency. Moreover, because the input impedance of the patch is very high, the impedance transformer (such as the $1 / 4 \lambda$ impedance transformer) is required to match the impedance. When it fabricated as a patch array antenna, the feed line and the impedance transformer become very complex and furthermore unwanted radiation from the feed network readily occurred. Thus, the radiation efficiency of a patch antenna is degraded. In the case of a MEMS aperture coupled antenna, because the feed line network and the radiation patch are implemented at the different substrates, the feed line network and the radiation patch can be optimized at the same time [8]. In other words, the radiating patch can be formed on the substrate with low dielectric constant and the feed line on the substrate with high dielectric constant. However, since it uses two substrates, two substrates have to be aligned exactly to achieve good performance. It is very difficult to align the substrates exactly and small misalignment results in large performance degradation at mm-wave frequency.

In this letter, a CPW-fed post-supported patch antenna using micromachining technology, which does not require substrate alignment or substrate etching, is presented at a mm-wave frequency of $60 \mathrm{GHz}$.

\section{Proposed Antenna Structure}

The structure of the proposed antenna is shown in Fig. 1. As shown in Fig. 1, the antenna is comprised of CPW feed line, a feeding post, two supporting posts, and a radiating patch. In the proposed antenna, the CPW line is used as a feed line. The CPW line shows better performance than the microstrip line at $\mathrm{mm}$-wave frequency. Because the ground planes are located in the vicinity of the signal line, the electromagnetic 
field can be guided better. Moreover, the geometry as the characteristic impedance is fixed in microstrip transmission line depending on the substrate properties and thus it can be difficult to implement microstrip line on some substrates, especially semiconductor substrates, at mm-wave frequency. However, in the case of CPW line, because the width of the signal line and the gap between the signal line and the ground can be adjusted having the same characteristic impedance, the proper geometry of CPW line can be selected. Also, back side via hole and substrate lapping process is not required in CPW line [9], [10]. Moreover, the ground planes are placed on the same surface of the signal line in CPW line. Therefore, when the patch is supported with a feeding post like coaxial probe feeding method, the ground planes of CPW feed line can be used as a ground plane of the radiating patch as shown in Fig. 1. The CPW feed line is on the substrate with high dielectric constant, but the radiating patch is formed on the air. Therefore, the radiating patch and the feed line can be optimized separately in the proposed antenna-like aperture coupled antenna. Because the patch is supported with metal posts on the air, there are no dielectric losses and thus the performance of the antenna can be improved. Also, the proposed antenna can be implemented on a single substrate and thus it does not require the accurate alignment of the substrates like aperture coupled antenna. Since it does not require substrate alignment or substrate etching, it is expected that it becomes the better candidate in manufacturing mm-wave antennas.

In the proposed antenna, the patch is supported with a feeding post and two additional supporting posts which are used just to support the patch more robustly. Because two additional posts are located at the virtual ground of the patch which is the center of E-plane, the additional supporting posts do not affect the electric performance of the antenna. When it is designed as a patch array antenna, it can be designed easily with a simple feed line network. Because an appropriate input impedance of the patch antenna can be selected by locating feeding post as shown in (1), which is the same with coaxial probe feeding method, the antenna can be directly matched to various input impedance. Thus, the $1 / 4 \lambda$ impedance transformer which is used in the edge feeding method is not required.

$$
R_{i n} \approx \frac{1}{2\left(G_{s}-G_{m}\right)} \cos ^{2}\left(\beta L_{1}\right) \Omega
$$

where $G_{s}$ is conductance at the radiating edge, $G_{m}$ is mutual conductance between the radiating edges, $L_{1}$ is the distance from the radiating edge, and $\beta$ is the propagation constant [11].

Moreover, the proposed antenna is easily integrated with MMICs such as VCOs and power amplifiers because no additional transitions, which are required in a horn antenna, are needed. Therefore, a single chip millimeter wave system including the antenna can be achieved, which has a very compact size, low cost, and high performance.

\section{Design AND FAbrication of the ANTENNA}

The proposed antenna is designed and optimized with a three-dimensional (3-D) EM simulator of CST Microwave

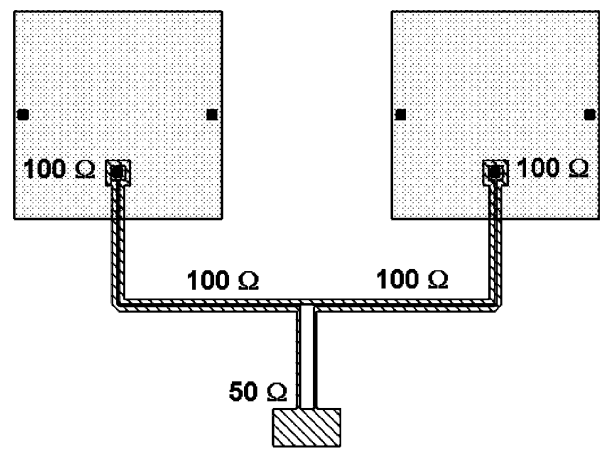

Fig. 2. Design of $2 \times 1$ patch array antenna.

Studio 4.3. A single patch antenna is directly matched with a $50-\Omega$ CPW feed line to a $50-\Omega$ input impedance which is the impedance of the measurement setup. Fig. 2 shows the designed $2 \times 1$ patch array antenna. The two patches are separated with the distance of $0.8 \lambda$ in which the antenna shows high gain property. As shown in Fig. 2, the input impedance of each patch is selected to $100 \Omega$ to design without $1 / 4 \lambda$ impedance transformer which is required in edge-fed patch antenna. Two patches are fed with a $100-\Omega$ CPW line and then two $100-\Omega$ CPW lines are combined with a $50-\Omega$ CPW line directly. Because there is no impedance transformer, the patch array antenna can be designed simply and minimize the discontinuities of the feed line which occur, such as the unwanted radiation. The simulated antenna gain and radiation efficiency of single patch antenna are $8.7 \mathrm{dBi}$ and $96 \%$ at $60 \mathrm{GHz}$, respectively. In the case of a $2 \times 1$ patch array antenna, the simulated antenna gain and the radiation efficiency are $9.9 \mathrm{dBi}$ and $94 \%$, respectively.

CPW-fed post-supported MEMS antenna is fabricated on the glass substrate of Corning 7740 which has the substrate thickness of $800 \mu \mathrm{m}$ and the dielectric constant of 4.6. The copper is used for metallization. At first, the bottom seed metal of $\mathrm{Ti} / \mathrm{Cu}$ is evaporated. The feed line of the antenna is patterned with the thick photoresist of AZ9260 and then electroplated with copper of $10-\mu \mathrm{m}$ thickness. After removing the photoresist, two-step coating process is performed to form the posts of the antenna with the thick photoresist of THB151N. The first coating is performed on the substrate with the thickness of about $100 \mu \mathrm{m}$ and then the posts are patterned with UV exposure and the development of photoresist. The posts are electroplated with copper, which are the almost filled by the thickness of $100 \mu \mathrm{m}$. After the posts with the thickness of $100 \mu \mathrm{m}$ are formed, the second coating is performed with the same thickness of the first coating or $100 \mu \mathrm{m}$. The posts are electroplated with copper which has the thickness of $100 \mu \mathrm{m}$. The total thickness of THB $151 \mathrm{~N}$ is about $200 \mu \mathrm{m}$ which is the height of the posts. After the post is formed, the seed metal of $\mathrm{Cu}$ is evaporated to form the radiating patch. The photoresist of AZ9260 is coated and patterned to make the radiating patch and then it is electroplated with copper of $10 \mu \mathrm{m}$ thickness. After the radiating patch is formed, AZ9260 is developed and the seed metal of the radiating patch is etched with $\mathrm{Cu}$ etchant and then THB151N is stripped. When THB151N is removed completely, the seed metal of feed line is removed clearly. Finally, 


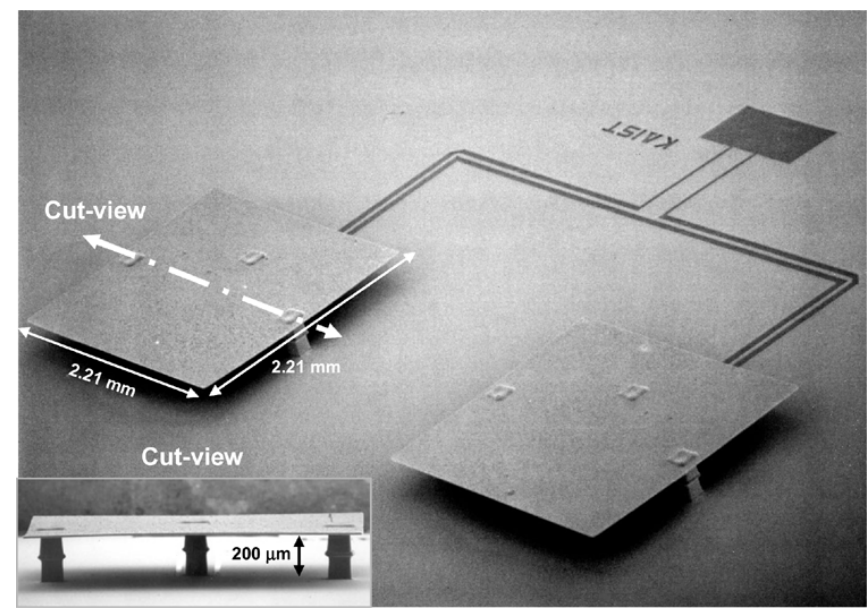

Fig. 3. Fabricated $2 \times 1$ patch array antenna.

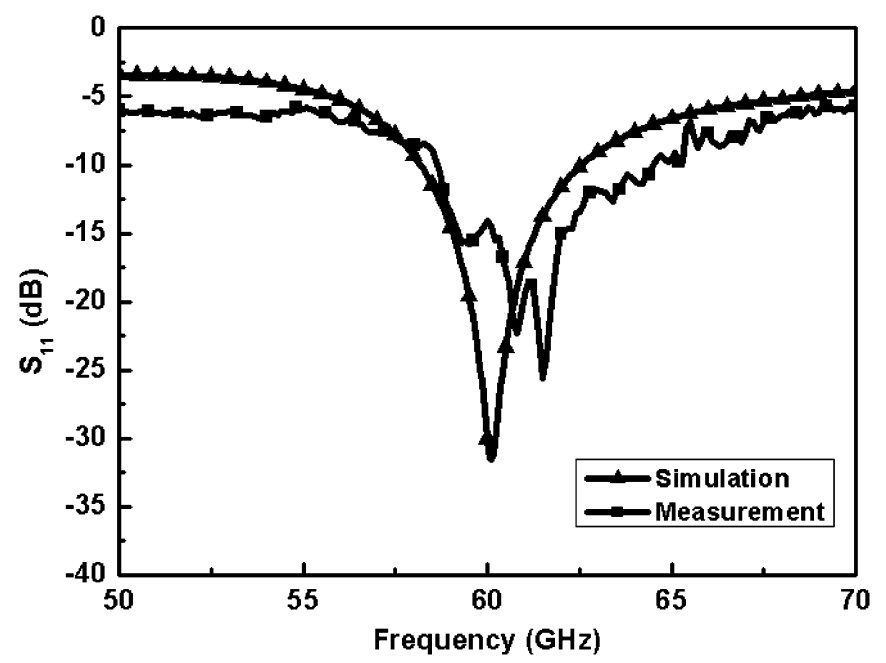

Fig. 4. Measured input return loss of single patch antenna.

the proposed MEMS antenna is formed, which is supported by the posts with the thickness of $200 \mu \mathrm{m}$. The whole fabrication process is compatible with commercial CMOS process. Fig. 3 shows the SEM image of the fabricated antenna. The size of $2 \times 1$ patch array antenna is $6.2 \mathrm{~mm} \times 4.2 \mathrm{~mm}$ and the radiating patch is $2.21 \mathrm{~mm} \times 2.21 \mathrm{~mm}$.

\section{MeAsurement Results}

Input return losses of the fabricated antenna are measured with Anritsu broadband vector network analyzer of ME7808A. All the measurement is performed on-wafer measurement on Karl Suss probe station. Fig. 4 shows the simulated and measured results of input return losses of the fabricated single patch antenna. $-10 \mathrm{~dB}$ bandwidth of the antenna is $5.8 \mathrm{GHz}$ from $58.7 \mathrm{GHz}$ to $64.5 \mathrm{GHz}$. Fig. 5 shows the simulated and measured results of input return losses of $2 \times 1$ patch array antenna. $-10 \mathrm{~dB}$ bandwidth of the antenna is $8.7 \mathrm{GHz}$ from $56.3 \mathrm{GHz}$ to $65 \mathrm{GHz}$. All the measurement results agree well with the simulation results.

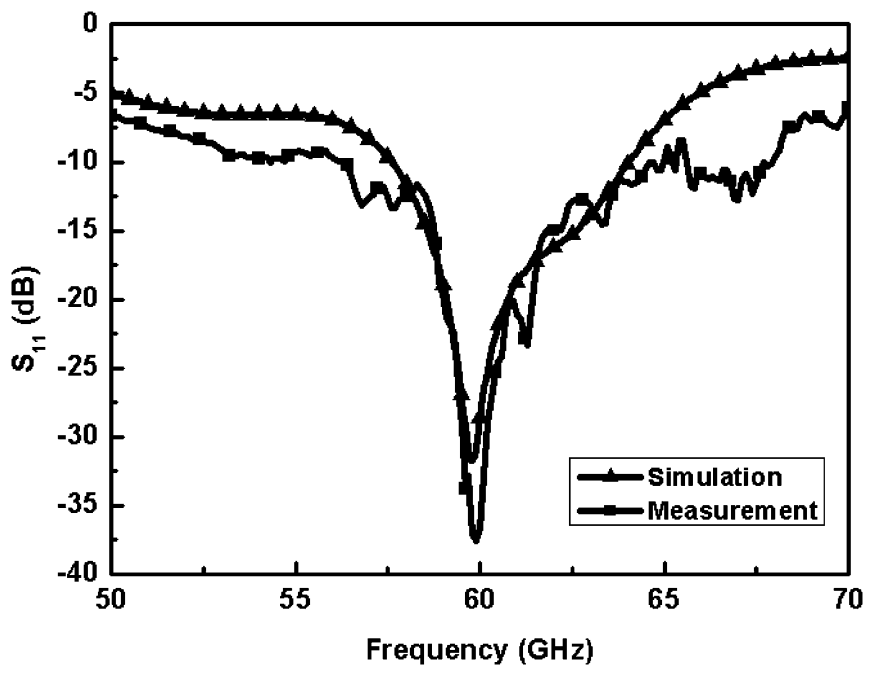

Fig. 5. Measured input return loss of $2 \times 1$ patch array antenna.

\section{CONCLUSION}

We have designed and fabricated a $60-\mathrm{GHz}$ CPW-fed postsupported patch array antenna using micromachining technology which is fully compatible with commercial CMOS process. The proposed antenna allows optimizations of the radiating patch and the feed line, respectively, on a substrate. Because the antenna is supported in the air, it shows broadband characteristics. Also, $2 \times 1$ patch array antenna without $1 / 4 \lambda$ impedance transformer is designed with a simple feed network. Because the proposed antenna allows the integration with MMICs, it can be applied for the system on a chip (SOC) including an antenna at mm-wave frequency.

\section{REFERENCES}

[1] S. Reynolds, B. Floyd, U. Pfeiffer, and T. Zwick, "60 GHz transceiver circuits in SiGe bipolar technology," in ISSCC Dig. Tech. Papers, Feb. 2004, pp. 442-538.

[2] C. H. Doan, S. Emami, A. M. Niknejad, and R. W. Brodersen, "Design of CMOS for $60 \mathrm{GHz}$ applications," in ISSCC Dig. Tech. Papers, Feb. 2004, pp. 440-538.

[3] C. H. Doan, S. Emami, D. Sobel, A. M. Niknejad, and R. W. Brodersen, "60 GHz CMOS radio for Gb/s wireless LAN," in IEEE RFIC Symp. Dig., Feb. 2004, pp. 225-228.

[4] G. P. Gauthier, A. Courtay, and G. M. Rebeiz, "Microstrip antennas on synthesized low dielectric-constant substrates," IEEE Trans. Antennas Propag., vol. 45, no. 8, pp. 1310-1314, Aug. 1997.

[5] G. P. Gauthier, J. P. Raskin, L. P. B. Katehi, and G. M. Rebeiz, "A 94-GHz aperture-coupled micromachined microstrip antenna," IEEE Trans. Antennas Propag., vol. 47, no. 12, pp. 1761-1766, Dec. 1999.

[6] J. P. Papapolymerou, R. F. Drayton, and L. P. B. Katehi, "Micromachined patch antennas," IEEE Trans. Antennas Propag., vol. 46, no. 2, pp. 275-283, Feb. 1998.

[7] B. Pan, Y. Yoon, P. Kirby, J. P. Papapolymerou, M. M. Tenzeris, and M. Allen, "A W-band surface micromachined monopole for low-cost wireless communication systems," in IEEE MTT-S Int. Dig., Jun. 2004, pp. $1935-1938$.

[8] D. M. Pozar, "Microstrip antenna aperture-coupled to a microstrip line," Electron. Lett., vol. 21, no. 1, pp. 49-50, Jan. 1985.

[9] K. J. Herrick, T. A. Schwarz, and L. P. B. Katehi, "Si-micromachined coplanar waveguides for use in high-frequency circuits," IEEE Trans. Microw. Theory Tech., vol. 46, no. 6, pp. 762-768, Jun. 1998.

[10] C. Schollhorn, Z. Weiwei, M. Morschbach, and E. Kasper, "Attenuation mechanisms of aluminum millimeter-wave coplanar waveguides on silicon," IEEE Trans. Electron Devices, vol. 50, no. 3, pp. 740-746, Mar. 2003.

[11] R. Grag, P. Bhartia, I. Bahl, and A. Ittipiboon, Microstrip Antenna Design Handbook. Norwell, MA: Artech House, 2000. 